\title{
Heading-determination using the sensor-fusion based maritime PNT Unit
}

\author{
Zhen Dai, Ralf Ziebold, Alexander Born, Evelin Engler \\ German Aerospace Center (DLR) \\ Neustrelitz, Germany
}

\begin{abstract}
This paper presents the heading determination technique with the concept of DLR maritime PNT Unit. By analyzing the characteristics of individual existing heading sensors, a sensor-fusion based heading-determination system according to the concept of DLR maritime PNT Unit is introduced. This system improves the accuracy as well as the continuity of the heading and attitude results compared to the individual sensors. The preliminary results from a measurement campaign are presented to evaluate the performance of individual sensors and the PNT Unit.
\end{abstract}

\section{INTRODUCTION}

In 2006, the member states of the International Maritime Organization (IMO) have started the "e-Navigation Initiative", whereby e-Navigation is defined as: "the harmonised collection, integration, exchange, presentation and analysis of maritime information onboard and ashore by electronic means to enhance berth to berth navigation and related services, for safety and security at sea and protection of the marine environment"'[1]. In a first step, user needs such as "improvement of reliability" and "indication of reliability" have been identified [2]. The concept of shipside PNT Unit has been developed to satisfy these user needs with respect to the onboard provision of position navigation and timing data (PNT).
Currently, vessels subject to the International Convention for the Safety of Life at Sea (SOLAS) [3] can either use single sensors to provide the PNT parameter (e.g. position, heading, speed over ground) individually or use an Integrated Navigation System (INS) [4].

Figure 1(a) represents the single sensor approach. Each sensor is described by an associated performance standard. The associated PNT data will be generated and displayed via an interface to applications. The onboard staff has to fuse the information from the different sensors. In the current INS approach, the sensors deliver their individually determined PNT output data to a shipboard processing layer, which is illustrated in Figure 1(b). The INS is performing plausibility checks on the incoming data and consistency checks on different sensors. Integrity is expected, if plausibility and consistency checks are passed [5]. For instance, the integrity monitoring of heading requires a comparison with a second heading sensor and a comparison with Course Over Ground (COG) information from another sensor. However, the two heading sensors might have common failure modes and hence it is unlikely to detect all possible failures by comparing both sensors. Therefore, the reliability of the INS output can not be guarantied even if the plausibility and consistency checks are passed. (a) Single Sensor Approach

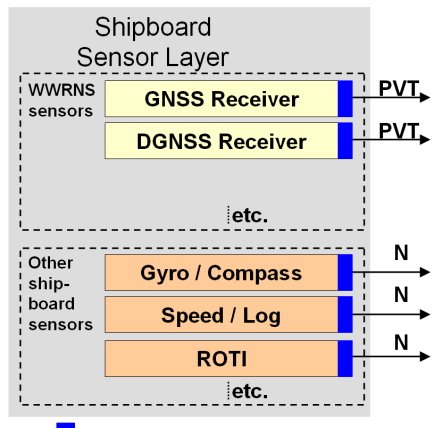

interface, point of type approval (b) Current INS Approach

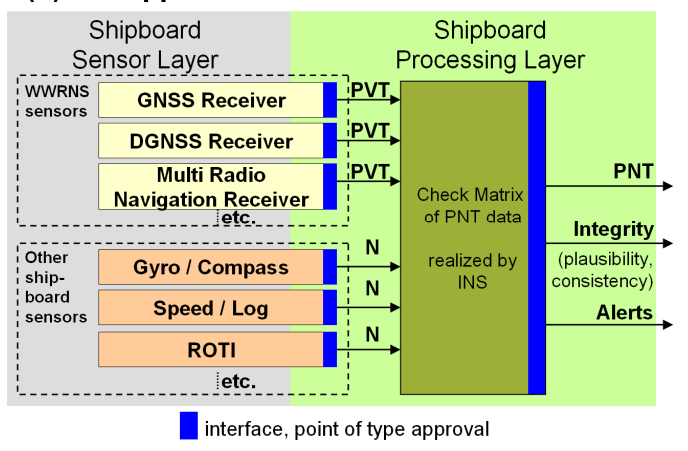

(c) PNT Unit Approach

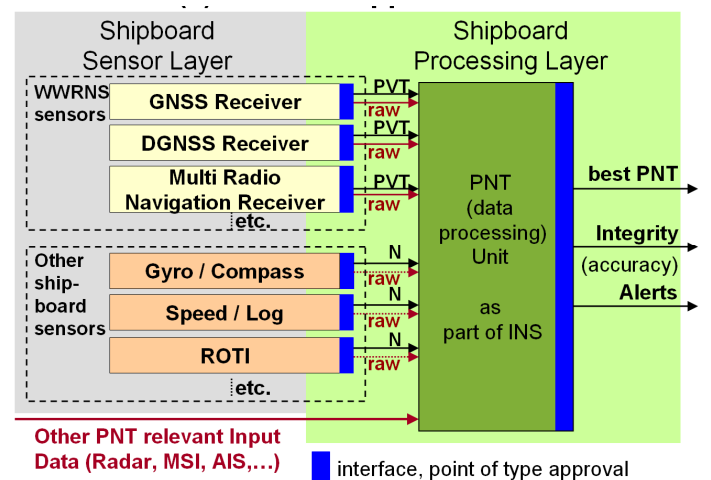

Figure 1 Concepts of single-sensor approach (a), current INS approach (b) and PNT Unit approach (c) 
In order to overcome these problems, a PNT data processing unit is introduced into the shipboard processing layer of the future INS, as illustrated in Figure 1(c). By means of sensor fusion techniques, the PNT Unit integrates all available PNT data and if available, also the raw data from the onboard sensors in order to provide the optimal PNT output data. The main advantage of this approach is the capability of improved integrity monitoring by using the sensor fusion techniques. Finally, integrity information for each PNT output data is generated based on the error estimation technique (see also [6]).

The introduction of the PNT Unit concept needs the definition of performance requirements. However, the current standards only refer to single sensors, and hence a specification of the PNT output parameters (e.g. position, velocity, attitude) is required by the concept of PNT Unit. As a basis for these definitions, the specification of maritime GNSS-based positioning service could be used. According to this, the quality of the output parameter should be evaluated by accuracy, integrity, continuity and availability for different operational areas (e.g. ocean, coast, port).

This paper concentrates on the determination of the heading angle, which is one of the output data of the PNT Unit. The heading angle is furthermore a basis for the definition of a Consistent Common Reference System (CCRS). Due to the size of vessels and the distribution of sensors, the position and velocity information measured by different sensors need to be converted to a common reference point. Heading information, as well as the other Euler angles and their change rates are needed for this conversion. Therefore, an accurate determination of heading is not only of great importance for itself but also for the other PNT parameters. Beside that, the integrity of the other output parameters like position and velocity relies also on the integrity of the attitude information.

In the remainder of the paper, we will first present the performance of individual maritime heading-determination sensors, together with the introduction of the common reference system and a short description of the realized measurement campaign. After that, the heading determination will be formulated and corresponding results will be given to show the performance improvement.

\section{MARITIME NORTH-FINDING SENSOR}

In maritime navigation, the north can indicate true north or magnetic north. True north refers to the earth rotation axis, whereas the magnetic north is defined by the horizontal component of the magnetic field of the earth [5]. North-finding can also be termed as heading determination or yaw angle determination.

In literature and in practice, various sensors are known to determine heading information of a vessel in maritime applications. The SOLAS directive differs between magnetic, gyrocompass and Transmitting Heading Device (THD) [3]. In this document, the carriage requirement, accuracy parameters and some other related requirements are already identified.
Besides the standard maritime sensors, other sensors have also been applied in the maritime navigation. For the development of an improved PNT Unit, the Inertial Measurement Unit (IMU) and GNSS-compass are taken into account.

\section{A. Consistent common reference system}

The heading determination refers to a specific body coordinate system. For example, the heading obtained from GNSS-compass reflects the deviation angle between a specific antenna baseline and the true north, whereas the IMU heading is then based on the X-axis of the IMU body frame. In order to unify and compare different heading sources, a CCRS is needed. In this paper, the ship body frame is chosen as the CCRS for the heading and attitude results. In the following discussions, without specific statement, the heading reflects the deviation angle between the ship longitudinal axis and the true north. For the conversion of heading onto common reference system, the following information should be available:

- For GNSS-compass: the coordinates of the GNSS antennas needs to be known within the ships coordinate system.

- $\quad$ For IMU: The attitude of IMU must be initialized with respect to the ship body frame. The related techniques will be detailed later in the paper.

- For gyrocompass: The gyrocompass should be well aligned with ship body frame, however, a small misalignment might occur, especially when gyrocompass has worked for a long term without proper settlement. The misalignment can be determined with the help of other heading-determination system. For example, if the GNSS-compass can be precisely converted to the ship body frame, then the heading of ship measured by GNSS-compass can be used to determine the misalignment angle of gyrocompass.

\section{B. Measurement campaign}

In order to collect test data for the development and test of the PNT Unit, first measurement campaigns have been performed in cooperation with the Federal Maritime and Hydrographic Agency (BSH) on the survey and research vessel DENEB. The vessel was equipped with three GNSS antennas and receivers (Javad Delta), an IMU (iMar IVRU FCAI), a gyrocompass, a Doppler speed log, an electromagnetic speed $\log$ and other standard shipborne sensors. Figure 2 shows the vessel DENEB, where the red circles mark the positions of the three GNSS antennas and the yellow circle indicates the position of the IMU installed near the centerline inside the vessel. 


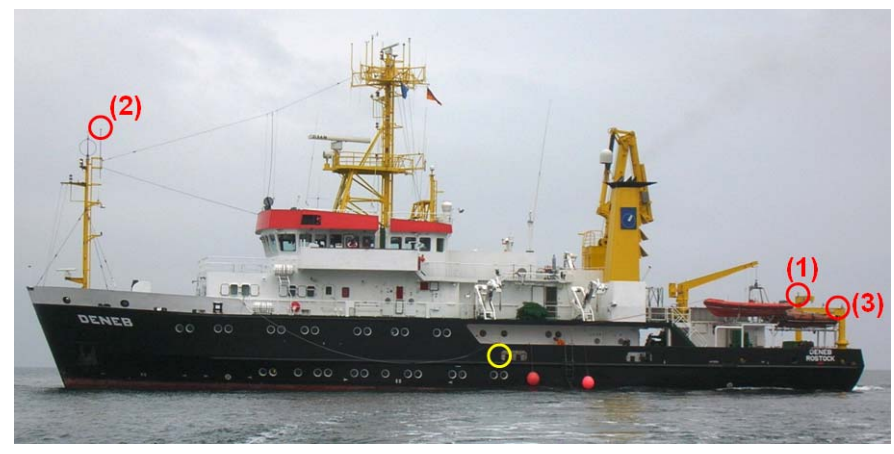

Figure 2 DENEB ship and GNSS antenna distribution

Most of the results presented in the paper are based on the data collected on 5th July 2011 from 10:00 to 11:00 (local time) near the port of Rostock. During this period, the vessel just departed from the port and starts a 7-day trip. The trajectory of the vessel is shown in Figure 3. Leaving the Warnow River, the vessel performed an anti-clockwise turning maneuver and finally it left the port and led into the Baltic Sea.

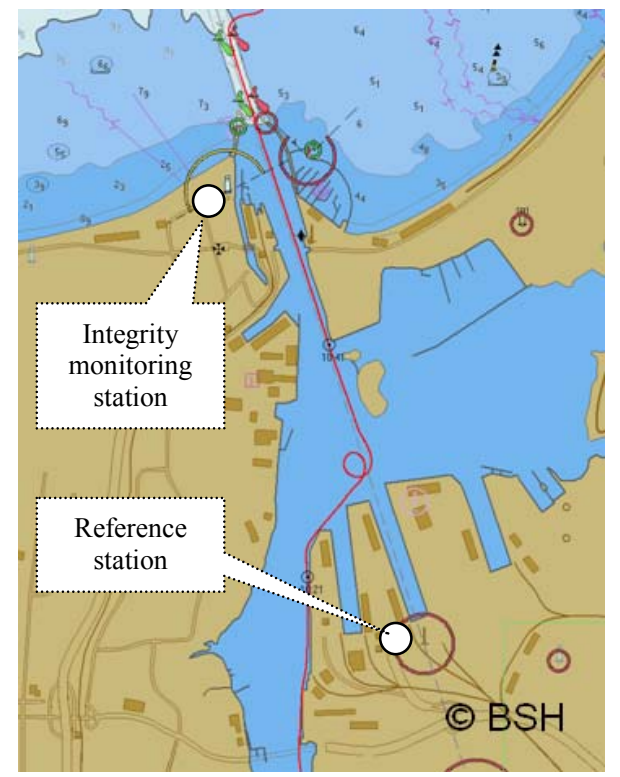

Figure 3 Trajectory of DENEB ship for one-hour trip in port area

In previous projects, DLR has developed and deployed a Maritime Ground Based Augmentation System (MGBAS) in the Rostock port. The MGBAS enables the position determination with decimeter accuracy and the monitoring of the GNSS signal qualities [8]. Using the MGBAS as well as the raw data from the IGS stations nearby, RTK results can be generated.

\section{Sensors}

\section{1) GNSS-compass}

Attitude information can be calculated from multiple onboard GNSS antennas. The baseline vector between antennas should be estimated using double-differenced carrier phase data. For two antennas, the yaw (heading) and pitch angles can be obtained. For three or more antennas, three-dimensional attitude information can be obtained. The accuracy is highly depended on the baseline length. Another implementation of GNSS compass can be done by using RTK positioning with the aid of a base station. This technique needs the accurate position of each antenna individually. It is usually used as reference data in post-processing and only applicable under the signal coverage of the RTK base station. In practice, the onboard approach would be preferred as it can also be used on open sea where no shore-side RTK-service is available.

The accuracy of a GNSS-compass is limited and strongly influenced by multipath effects, ephemeris, clock errors (satellite/receiver), ionospheric and tropospheric conditions. Another potential problem in practice lies in the montage of the antennas. The GNSS-compass is based on a rigid antenna body frame, i.e. the baseline length between antennas should keep unchanged. However, in the maritime navigation, due to the unpredictable weather and wave condition, due to the complexity of the antenna montage, due to the ship maneuver, the antenna baseline might present slight changes. This will cause errors to the attitude results.

In order to show the performance of GNSS-compass, a 70minute data collected starting from local time 0:00 on July-52011 is used when the ship is docked at the docking position. The heading results estimated by GNSS-compass are depicted in the upper figure of Figure 4, where the red star indicates the epochs at which GNSS-compass does not output reliable results. The quality of the GNSS-compass can be evaluated by the baseline length. The estimated baseline length should keep unchanged as long as the GNSS carrier phase measurements are correctly processed. The between-epoch variations of estimated baseline length are presented in the lower figure.
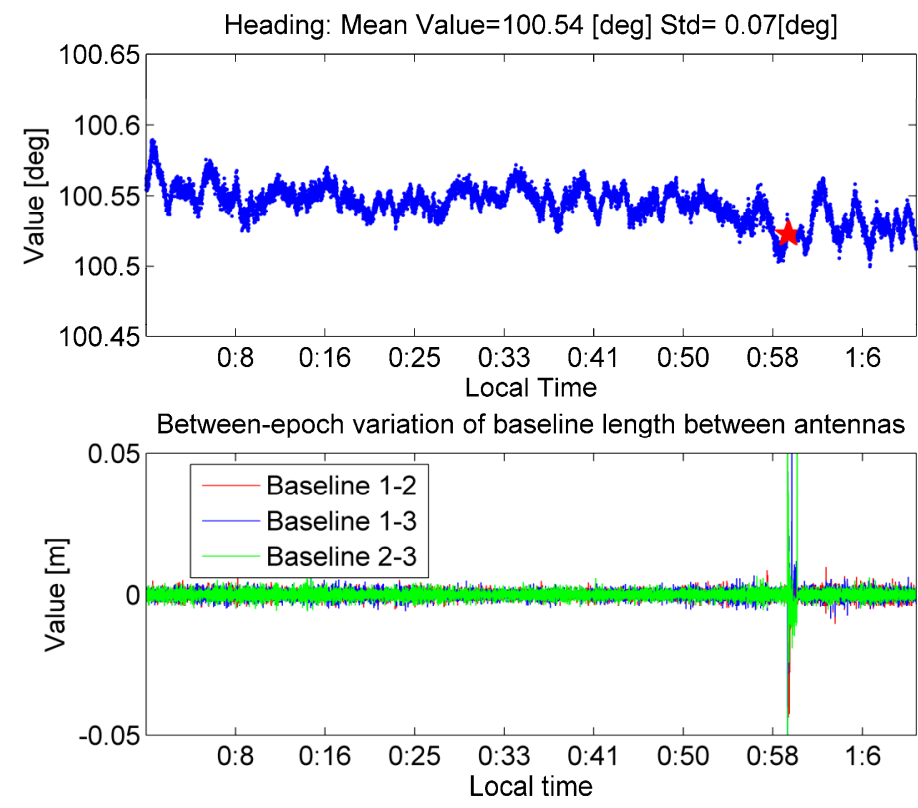

Figure 4 Heading determination using GNSS-compass in quasi-static scenario at port

Concerning the upper figure, the unavailable results are mainly caused by the failed solution of integer ambiguities of carrier phase measurements. The number of unavailable epochs takes about $0.5 \%$ of total epochs in this data set. In 
order to detect the outage of GNSS-compass, integrity monitoring should be performed. Beside that, a backup system is needed to bridge the outage of GNSS-compass.

From the lower figure it can be seen that, once the ambiguity resolution of carrier phase fails, the baseline length will change with a magnitude of several centimeters. Without integrity monitoring, this will bring significant errors to the attitude results. From this aspect, the checks on the variation of baseline length can also serve as the test of proper operation of the GNSS-compass.

\section{2) $I M U$}

An IMU is composed of three gyroscopes for angular rate measurements and three accelerometers for acceleration measurements. As long as the static biases of gyroscopes and accelerometers can be well estimated, the IMU can measure the vehicle dynamics with high accuracy for short-time operation period. With the strapdown processing, the angular rate and acceleration measurements can be numerically integrated to yield the attitude, velocity and position. However, the initial values for these parameters have to be determined before performing the strapdown processing. Concerning the IMU attitude, commonly-used initialization techniques include the self-alignment and the use of other sensors. The self-alignment aims at the attitude determination by IMU itself in a static or quasi-static situation. The initialization technique based on the external sensors usually needs the conversion onto the IMU body frame. This conversion is, on the other hand, based on the IMU selfalignment. Therefore, the following text focuses on the IMU self-alignment.

The IMU self-alignment comprises two processes: (1) leveling which calculates roll and pitch angles, and (2) gyrocompassing which determines the heading angle. Leveling is based on the fact that if the IMU is stationary or moves with constant velocity, the only specific force sensed by the accelerometers is the reaction to gravity. The principle behind gyrocompassing is that, when IMU is stationary, the only rotation it senses is the earth rotation [9]. The IMU selfalignment can be formulated as:

$$
\begin{aligned}
& p=\arctan \left(f_{x} / \sqrt{f_{y}^{2}+f_{z}^{2}}\right) \\
& r=\arctan \left(-f_{y} /-f_{z}\right) \\
& y=\frac{-\omega_{y} \cos r+\omega_{z} \sin r}{\omega_{x} \cos p+\omega_{y} \sin r \sin p+\omega_{z} \cos r \sin p}
\end{aligned}
$$

where $p, r$ and $y$ represent pitch, roll and yaw (also heading), respectively; $f$ stands for the acceleration measurement; $\omega$ is the angular rate. The leveling (the upper and middle equations) should be applied prior to the gyrocompassing. In order to eliminate the stochastic bias of IMU raw data, the IMU raw data over a specific sampling length can be averaged at first and then used for the gyrocompassing. This is particularly important for the ships docked at the port area, as there might be slight wind and wave effects on the ships, leading to quasi- static situation. Figure 5 shows the standard deviation of the calculated heading results for different averaging times for both, really static measurements (in the laboratory) and quasistatic measurements from the vessel DENEB at the port in Rostock.

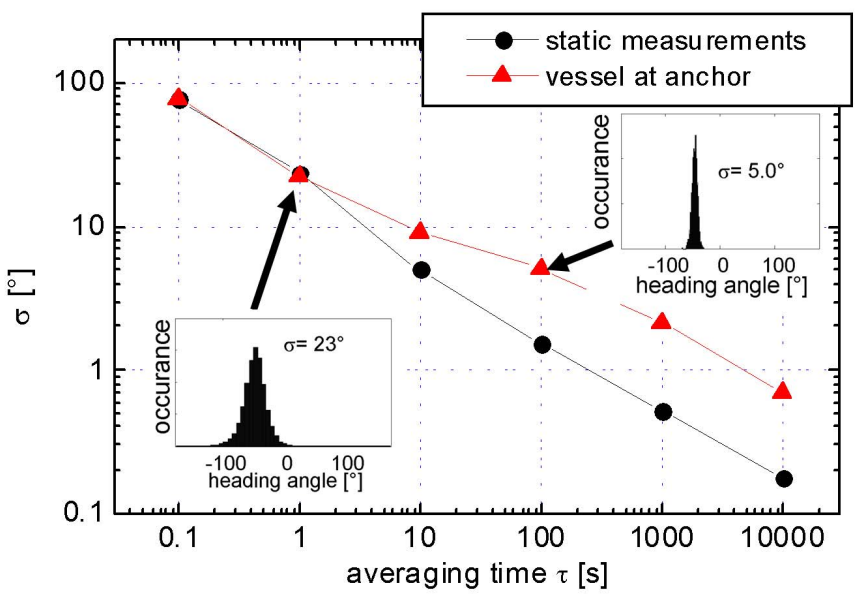

Figure 5 Gyrocompassing precision under different averaging length

It can be seen that longer averaging time can normally yield higher precision. For averaging times $\tau>1 \mathrm{~s}$, gyrocompassing for the quasi-static scenario leads to worse results compared to the real static scenario. However, even for the vessel at anchor, a precision with $\sigma<1^{\circ}$ can be achieved.

If a proper initialization is given to the IMU, the update of attitude, velocity and position results made by strapdown processing can be formulated as [9]:

Attitude update

$\mathbf{C}_{b}^{e}(+) \approx \mathbf{C}_{b}^{e}(-)\left(\mathbf{I}_{3}+\mathbf{\Omega}_{i b}^{b} \tau_{i}\right)-\mathbf{\Omega}_{i e}^{e} \mathbf{C}_{b}^{e}(-) \tau_{t}$

Specific-force frame transformation

$\mathbf{f}_{i b}^{e} \approx \frac{1}{2}\left(\mathbf{C}_{b}^{e}(-)+\mathbf{C}_{b}^{e}(+)\right) \mathbf{f}_{i b}^{b}$

Velocity update

$\mathbf{v}_{e b}^{e}(+) \approx \mathbf{v}_{e b}^{e}(-)+\left(\mathbf{f}_{i b}^{e}+\mathbf{g}_{b}^{e}-2 \mathbf{\Omega}_{i e}^{e} \mathbf{v}_{e b}^{e}(-)\right) \tau_{i}$

Position update

$\mathbf{p}_{e b}^{e}(+) \approx \mathbf{p}_{e b}^{e}(-)+\left(\mathbf{v}_{e b}^{e}(-)+\mathbf{v}_{e b}^{e}(+)\right) \tau_{i}$

where $b$ means the IMU body frame; $e$ denotes the ECEF frame; $i$ indicates the inertial frame; $\mathbf{C}_{b}^{e}$ is the Direction Cosine Matrix (DCM) from body frame to ECEF frame, $\boldsymbol{\Omega}$ is the skew-symmetric matrix for angular rate measurements; $\mathbf{f}_{i b}^{b}$ is the vector of acceleration measurements from the accelerometers; $\mathbf{g}$ is the gravity vector; $\mathbf{p}$ is the position; $\mathbf{v}$ is the velocity; $\tau$ is the IMU observation sampling interval; $\mathbf{I}$ is a identity matrix. The attitude update is the first step of the strapdown processing and its errors will be accumulated to the position and velocity results. As the paper focuses on the attitude aspects, only the attitude updates made by the 
strapdown processing will be presented. Using the GNSScompass as the initialization of IMU attitude and furthermore the reference values of attitude results, the attitude errors given by the IMU strapdown processing are presented in Figure 6.
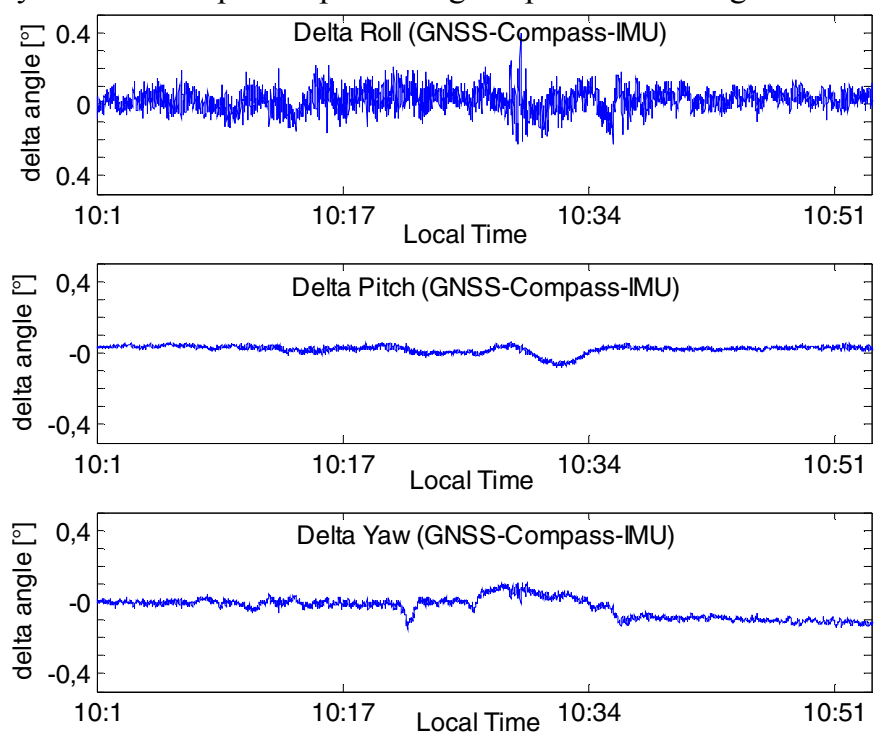

Figure 6 Error of IMU strapdown processing compared to the reference values obtained from GNSS-compass

It can be seen that, within one hour operation of strapdown processing, the IMU does not show significant offset with respect to the GNSS-compass. From this point of view, the IMU can serve as a backup system to the heading and attitude angles, in case of the failure of the major sensors.

\section{3) Gyrocompass}

The gyrocompass finds the orientation about the vertical axis (yaw/heading) by measuring the direction of Earth's axis of rotation relative to inertial space. The gyrocompass itself consists of a fast rotating, gimbal-mounted gyroscope which is using the effect of gyroscopic precession [10]. A gyrocompass is subject to following errors [10]: (1) steaming error: rapid changes in course and speed can cause a deviation. (2) Time dependent effects: the accuracy of the gyrocompass is only stable for short periods and requires an uninterruptable source of power and a warm-up period. (3) Errors when used at very high latitudes.

A first analysis of the error behavior of the onboard gyrocompass has been performed using the dynamic data from the measurement trip, where the GNSS compass acts as a reference. In Figure 7 and Figure 8, typical behavior of the gyrocompass error is illustrated. In the upper and middle graphs, the attitude angles roll, pitch and yaw (heading) are plotted. In the lower graphs the heading difference between the gyrocompass and GNSS compass is shown. In Figure 7, a slow drift of the gyrocompass error with a maximum value up to 1.5 degrees can be observed. Figure 8 shows data from another day, where at the end of scenario, larger rolling and pitching of the vessel due to moderate waves can be seen. Meanwhile, significant gyrocompass errors can be found during the change of the ship's course.
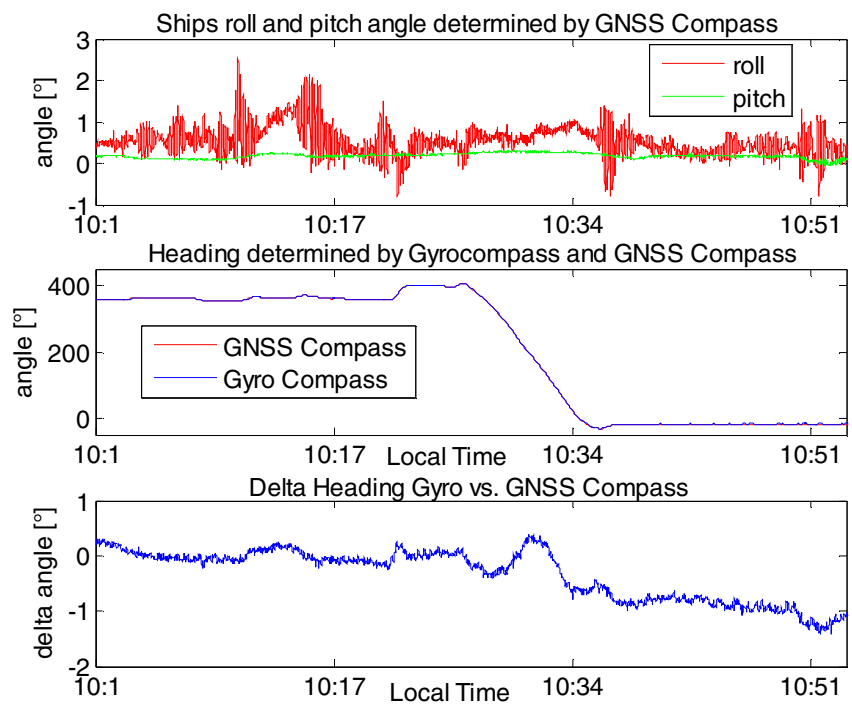

Figure 7 Ships attitude determined by GNSS Compass and Gyrocompass for a $1 \mathrm{~h}$ scenario at calm sea conditions
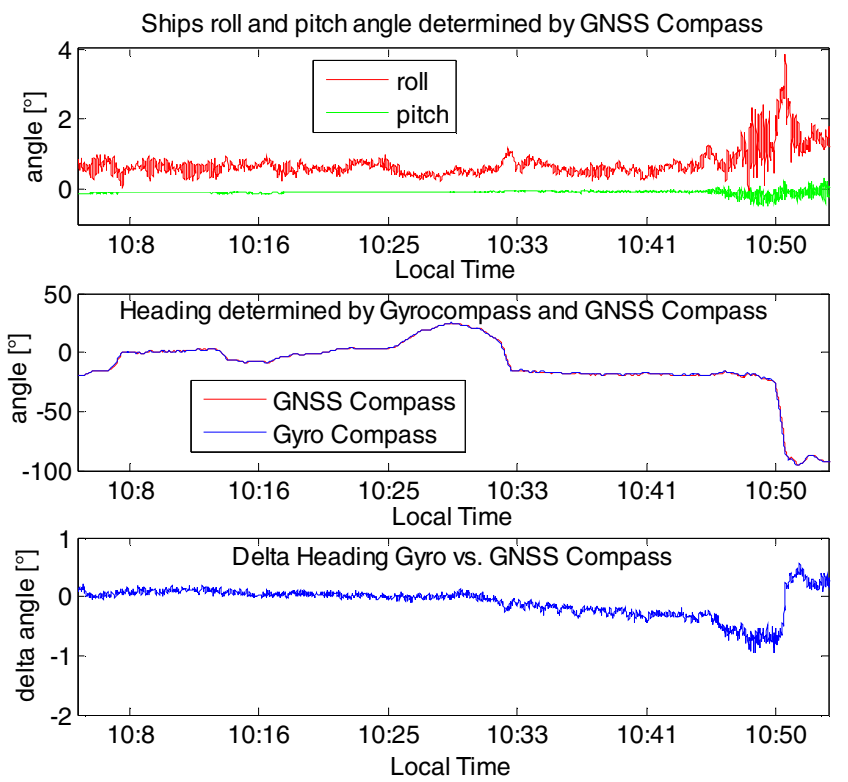

Figure 8 Ships attitude determined by GNSS Compass and Gyrocompass for a $1 \mathrm{~h}$ scenario at moderate sea conditions

\section{SENSOR FUSION}

In the former sections, the performance of different maritime north-finding sensors has been discussed. In order to overcome the shortcomings of individual sensors, the maritime PNT Unit integrates the data of these sensors with hybrid architecture. The GNSS pseudorange and Doppler measurements, as well as the output from north-finding sensors will serve as measurements. The IMU describes the dynamics. The 
measurement model and dynamic model will be integrated using an Extended Kalman Filter (EKF). In this section, the mathematic formulation and some preliminary results will be presented.

The routine of an EKF starts with the linearization of the dynamic and measurement models. At epoch $k$, the dynamic model is linearized around the a posteriori state estimates at last epoch (denoted by $\hat{\mathbf{x}}_{k-1}^{+}$) and the measurement model is linearized around the a priori state estimates at current epoch (denoted by $\hat{\mathbf{x}}_{k}^{-}$), so that the nonlinear relation $f(\cdot)$ and $h(\cdot)$ can be replaced by the Jacobian matrices $\mathbf{F}$ and $\mathbf{H}$, respectively. The EKF routine can be formulated as [11]:

\section{Filter initialization:}

$$
\begin{aligned}
& \hat{\mathbf{x}}_{0}^{+}=E\left(\mathbf{x}_{0}\right) \\
& \mathbf{P}_{0}^{+}=E\left[\left(\mathbf{x}_{0}-\hat{\mathbf{x}}_{0}^{+}\right)\left(\mathbf{x}_{0}-\hat{\mathbf{x}}_{0}^{+}\right)^{T}\right]
\end{aligned}
$$

\section{Time update:}

$$
\begin{aligned}
& \hat{\mathbf{x}}_{k}^{-}=f_{k-1}\left(\hat{\mathbf{x}}_{k-1}^{+}\right) \\
& \mathbf{P}_{k}^{-}=\mathbf{F}_{k-1} \mathbf{P}_{k-1}^{+} \mathbf{F}_{k-1}^{T}+\mathbf{Q}_{k-1}
\end{aligned}
$$

\section{Kalman gain calculation:}

$$
\mathbf{K}_{k}=\mathbf{P}_{k}^{-} \mathbf{H}_{k}^{T}\left(\mathbf{H}_{k} \mathbf{P}_{k}^{-} \mathbf{H}_{k}^{T}+\mathbf{R}_{k}\right)^{-1}
$$

\section{Measurement update:}

$$
\begin{aligned}
& \hat{\mathbf{x}}_{k}^{+}=\hat{\mathbf{x}}_{k}^{-}+\mathbf{K}_{k}\left[\mathbf{z}_{k}-h_{k}\left(\hat{\mathbf{x}}_{k}^{-}\right)\right] \\
& \mathbf{P}_{k}^{+}=\left(\mathbf{I}-\mathbf{K}_{k} \mathbf{H}_{k}\right) \mathbf{P}_{k}^{-}\left(\mathbf{I}-\mathbf{K}_{k} \mathbf{H}_{k}\right)^{T}+\mathbf{K}_{k} \mathbf{R}_{k} \mathbf{K}_{k}^{T}
\end{aligned}
$$

where $\mathbf{x}$ is the state vector; $f$ and $\mathbf{F}$ are the original and linearized dynamic matrix, $\mathrm{h}$ and $\mathbf{H}$ are the original and linearized design matrix, respectively; $\mathbf{P}$ is the state error covariance matix; $\mathbf{Q}$ is the dynamic error covariance matrix; $\mathbf{K}$ is the Kalman gain; $\mathbf{z}$ is the measurement vector; $\mathbf{I}$ is the identity matrix; $\mathbf{R}$ is the measurement error covariance matrix.

The error states of the Kalman filters in Earth-CenteredEarth-Fixed frame (ECEF) read [9]:

$$
\mathbf{x}_{I M U}^{e}=\left[\begin{array}{lllllll}
\delta \boldsymbol{\psi}_{e b}^{e} & \delta \mathbf{v}_{e b}^{e} & \delta \mathbf{r}_{e b}^{e} & \mathbf{b}_{a} & \mathbf{b}_{g} & \delta t & \delta \dot{t}
\end{array}\right]
$$

where $\psi$ reflects the attitude angles from the body frame to ECEF frame; $\mathbf{v}$ is the velocity vector expressed in ECEF; $\mathbf{r}$ is the position vector expressed in ECEF; $\mathbf{b}_{\mathrm{a}}$ and $\mathbf{b}_{\mathrm{g}}$ are biases of accelerometer and gyroscope, respectively, given in IMU body frame; $\delta t$ and its time derivative represent the receiver clock offset and receiver clock error rate.

With the strapdown processing, the IMU sensor errors are accumulated onto the navigation solutions. In order to quantify the errors brought by the IMU raw data, the system matrix is needed by each strapdown routine, as expressed by [9]:

$$
\begin{aligned}
& \dot{\mathbf{x}}=\mathbf{F}_{I M U}^{e} \mathbf{x}(-)+\mathbf{w} \\
& \mathbf{F}_{I M U}^{e}= \\
& \left(\begin{array}{cccccccc}
-\mathbf{\Omega}_{i e}^{e} & \mathbf{O}_{3} & \mathbf{O}_{3} & \mathbf{O}_{3} & \hat{\mathbf{C}}_{b}^{e} & 0 & 0 \\
{\left[-\left(\hat{\mathbf{C}}_{b}^{e} \hat{\mathbf{f}}_{i b}^{b}\right) \wedge\right.} & -2 \mathbf{\Omega}_{i e}^{e} & \frac{2 \mathbf{g}}{r_{e S}^{e}} \frac{\hat{r}_{e b}^{e}}{\left|\hat{r}_{e b}^{e}\right|^{2}} \hat{r}_{e b}^{e T} & \hat{\mathbf{C}}_{b}^{e} & \mathbf{O}_{3} & 0 & 0 \\
\mathbf{O}_{3} & \mathbf{I}_{3} & \mathbf{O}_{3} & \mathbf{O}_{3} & \mathbf{O}_{3} & 0 & 0 \\
\mathbf{O}_{3} & \mathbf{O}_{3} & \mathbf{O}_{3} & \mathbf{O}_{3} & \mathbf{O}_{3} & 0 & 0 \\
\mathbf{O}_{3} & \mathbf{O}_{3} & \mathbf{O}_{3} & \mathbf{O}_{3} & \mathbf{O}_{3} & 0 & 0 \\
0 & 0 & 0 & 0 & 0 & 0 & 1 \\
0 & 0 & 0 & 0 & 0 & 0 & 0
\end{array}\right) \\
& \mathbf{w}=\left[\begin{array}{cccccccc}
\mathbf{w}_{\omega} & \mathbf{w}_{f} & 0 & \mathbf{w}_{a} & \mathbf{w}_{g} & \mathbf{w}_{t} & \mathbf{w}_{i}
\end{array}\right]
\end{aligned}
$$

where $\mathbf{F}$ is the system matrix applied in the ECEF frame; $r_{e s}^{e}$ is the distance from the earth geometric center to the earth surface; $\mathbf{g}$ is the local gravity; $\hat{r}_{e b}^{e}$ is the position of the IMU in ECEF; the other terms are already identified in (2). The noise vector $\mathbf{w}$ contains, in the indicated order, gyroscope bias, acceleration bias, acceleration noise, angular rate noise, receiver clock error and receiver clock rate noise. These noise terms are described by the error covariance matrix $\mathbf{Q}$ in the Kalman filter routine:

$$
\mathbf{Q}_{I M U}=\operatorname{diag}\left(n_{r g}^{2} \mathbf{I}_{3} \quad n_{r a}^{2} \mathbf{I}_{3} \quad \mathbf{O}_{3} \quad n_{b g}^{2} \mathbf{I}_{3} \quad n_{b a}^{2} \mathbf{I}_{3} \quad n_{t}^{2} \quad n_{i}^{2}\right) \tau_{s}
$$

where $n$ represents power spectral densities of component of vector $\mathbf{w}$ given in (5).

In the classic GNSS/IMU tightly-coupled integration, the observation vector includes the GNSS pseudorange and Doppler data. In order to enhance the heading and attitude determination, the observation vector can also contain the heading information from GNSS-compass and gyrocompass, as well as the pitch and roll angles from GNSS-compass. This is an extension of the classic tightly-coupled GNSS/IMU integration architecture, making the heading (or attitude) information fully observable. The measurement vectors from different integration strategy can be expressed as:

$$
\begin{aligned}
& \tilde{\mathbf{z}}=\left[\begin{array}{llllllll}
p_{1} & p_{2} & \cdots & p_{n p} & d_{1} & d_{2} & \ldots & d_{n d}
\end{array}\right]^{T} \\
& \mathbf{z}_{1}=\left[\begin{array}{ll}
y_{\text {gyro }} & \tilde{\mathbf{z}}
\end{array}\right]^{T} \\
& \mathbf{z}_{2}=\left[\begin{array}{ll}
y_{G N S S} & \tilde{\mathbf{z}}
\end{array}\right]^{T} \\
& \mathbf{z}_{3}=\left[\begin{array}{llll}
y_{G N S S} & p_{G N S S} & r_{G N S S} & \tilde{\mathbf{z}}
\end{array}\right]^{T} \\
& \mathbf{z}_{4}=\left[\begin{array}{lllll}
y_{\text {GNSS }} & p_{\text {GNSS }} & r_{\text {GNSS }} & y_{\text {gyro }} & \tilde{\mathbf{z}}
\end{array}\right]^{T}
\end{aligned}
$$

where $\tilde{\mathbf{z}}$ is the observation vector of the classic GNSS/IMU integration which is composed of GNSS pseudorange and Doppler data; the subscripts $n p$ and $n d$ represent number of available pseudorange and Doppler measurements; $y, p$ and $r$ with subscripts "GNSS" indicate the attitude angles obtained from GNSS. Depending on the sensors available and application requirements, different heading or attitude information can be added into the measurement vector. Eq. (7) 
introduces four types of measurement expansion formulated from $\mathbf{z}_{1}$ to $\mathbf{z}_{4}$. These vectors indicate, respectively, the addition of (1) gyrocompass, (2) yaw angle from GNSS-compass, (3) three dimensional attitude angles from GNSS-compass (4) attitude angles of GNSS-compass together with the gyrocompass. It should be noted that the measurement vector of the classic IMU/GNSS integration $\tilde{\mathbf{z}}$ also exists in the expended measurement vector.

The measurement matrix $\mathbf{H}$ projects the measurements onto the state domain. In a classic GNSS/IMU integration, the $\mathbf{H}$ matrix associated to the measurement vector $\tilde{\mathbf{z}}$ in (7) and the state vector in (4) can be formulated as:

$$
\mathbf{H}=\left[\begin{array}{ccccccc}
O_{1 \times 3} & O_{1 \times 3} & h_{1} & O_{1 \times 3} & O_{1 \times 3} & 1 & 0 \\
O_{1 \times 3} & O_{1 \times 3} & h_{2} & O_{1 \times 3} & O_{1 \times 3} & 1 & 0 \\
\vdots & \vdots & \vdots & \vdots & \vdots & \vdots & \vdots \\
O_{1 \times 3} & O_{1 \times 3} & h_{n p} & O_{1 \times 3} & O_{1 \times 3} & 1 & 0 \\
O_{1 \times 3} & h_{1} & O_{1 \times 3} & O_{1 \times 3} & O_{1 \times 3} & 0 & 1 \\
O_{1 \times 3} & h_{2} & O_{1 \times 3} & O_{1 \times 3} & O_{1 \times 3} & 0 & 1 \\
\vdots & \vdots & \vdots & \vdots & \vdots & \vdots & \vdots \\
O_{1 \times 3} & h_{n d} & O_{1 \times 3} & O_{1 \times 3} & O_{1 \times 3} & 0 & 1
\end{array}\right]
$$

where $h$ is the line-of-sight vector describing the satellite position with respect to the GNSS antenna; $\mathbf{O}$ is the zerovector. A detailed expression can be found in [9]. In case of integration with other attitude or heading sensors, the $\mathbf{H}$ matrix should be modified accordingly. As the heading information is just loosely-coupled with the Kalman filter states, the $\mathbf{H}$ matrix can be expanded by giving the one-valued component at the corresponding position. For example, if GNSS-compass is integrated using the measurement vector $\mathbf{z}_{3}$ given in (7), the new matrix, termed as $\mathbf{H}_{3}$, becomes:

$$
\mathbf{H}_{3}=\left[\frac{\mathbf{I}_{3} \mathbf{O}_{3 \times 14}}{\mathbf{H}}\right]
$$

where the identity matrix I indicates the addition of GNSScompass.

The measurement error covariance matrix $\mathbf{R}$ can be determined by giving the a priori error of GNSS pseudorange and Doppler data. If the gyrocompass or GNSS-compass is integrated, the associated errors should also be defined in $\mathbf{R}$.

In maritime navigation, a common problem of the classic GNSS/IMU integration lies in the much weaker observability of heading (yaw) than pitch and roll angles. In other words, the roll and pitch errors of IMU might be identified and corrected by GNSS/IMU, however, the GNSS/IMU integration does not provide the same level sensitivity to detect the heading errors. This problem is particularly significant in maritime navigation due to the limited maneuverability of the vessel. In order to describe this problem, 5-degree errors are added to the initial attitude angles. The attitude results from a classic tightly-coupled GNSS/IMU integration with an execution length of 20 minutes are presented in Figure 9.
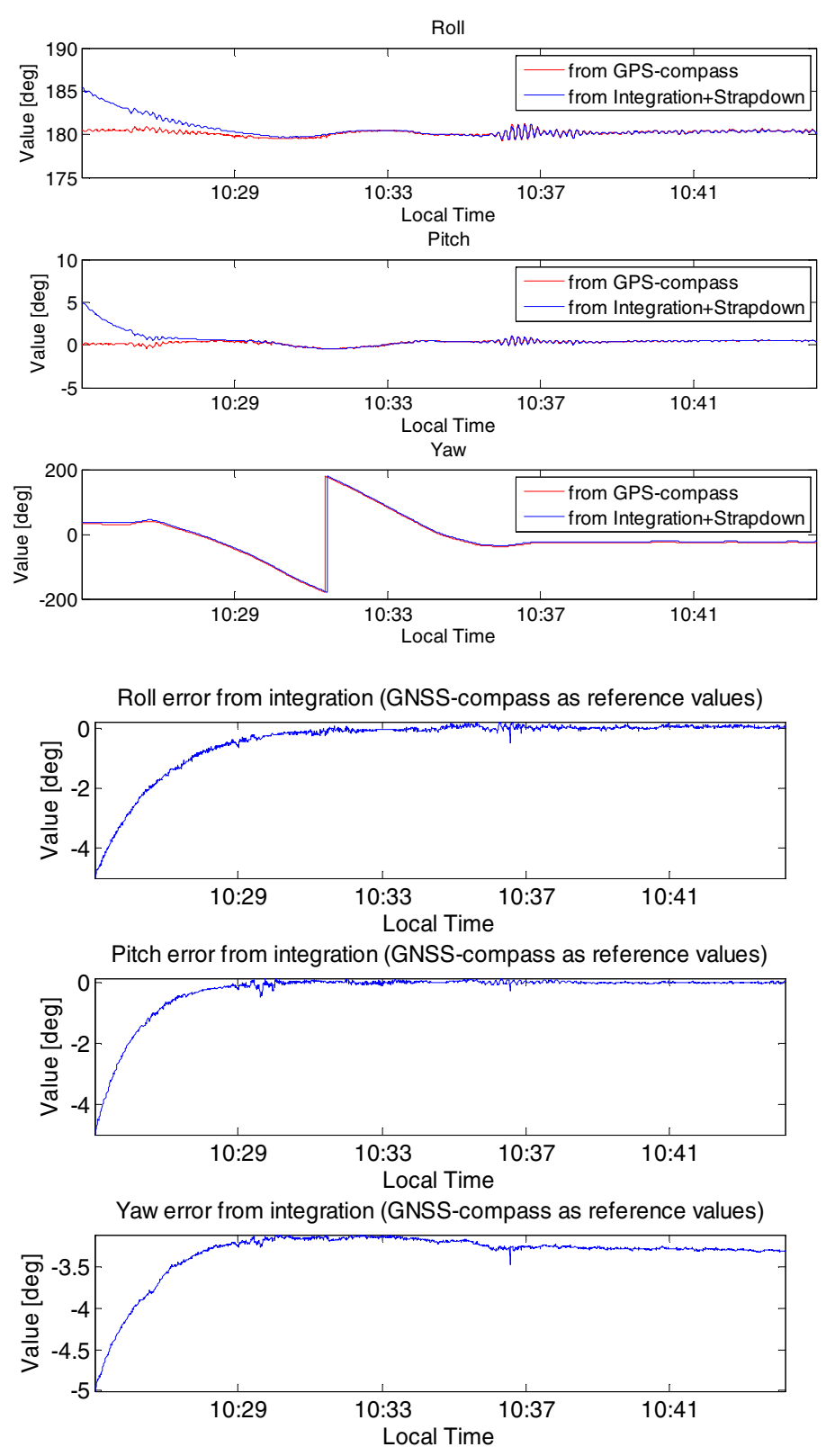

Figure 9 Attitude angles estimated by GNSS/IMU integration with initial attitude errors

In the upper figure, the attitude results obtained from GNSS/IMU integration and from GNSS-compass are depicted, respectively, where the latter are seen as the reference values. In the lower figure, the difference values between both attitude sources are presented in order to show the attitude errors from the GNSS/IMU integration. It can be seen that, the roll and pitch errors can be gradually adjusted, as the errors approach zero values after UTC time around 10:35 after several minutes convergence. However, the heading errors cannot be well observed and corrected. After 20 minutes, the heading shows still an offset about 3.5 degrees compared to the reference values. 
In order to overcome this problem, the heading should become observable by assigning direct measurement. To do this, either the gyrocompass or GNSS-compass or both together could be added into the measurement vector. In Figure 10 the results are shown for the same scenario as before but now with the additional usage of the gyrocompass within the measurement vector.

Roll error from integration (GNSS-compass as reference values)

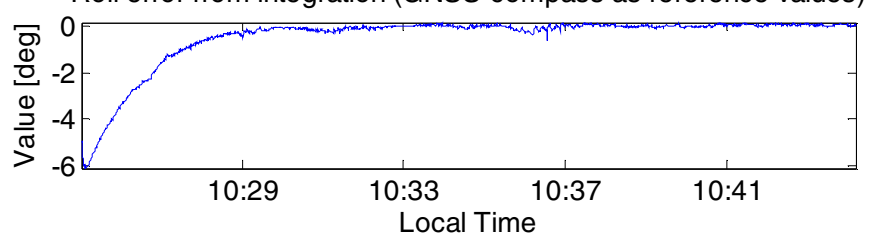

Pitch error from integration (GNSS-compass as reference values)

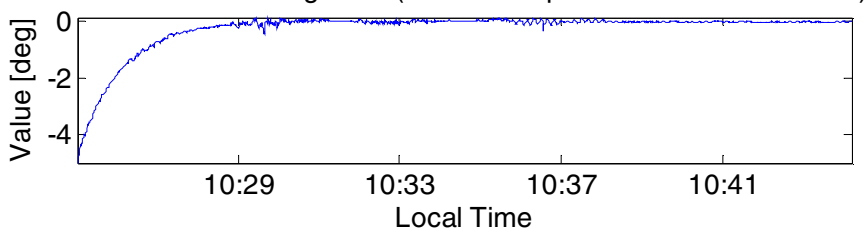

Yaw error from integration (GNSS-compass as reference values)

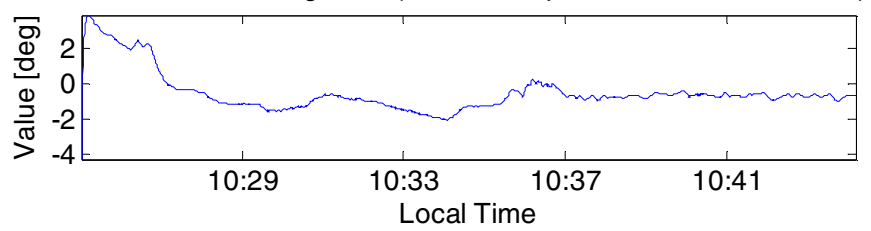

Figure 10 Attitude errors from GNSS+IMU+Gyrocompass integration

In comparison with Figure 9, the converging phase of pitch and roll angles still exist, whereas the uncorrected heading offset is almost eliminated. However, the gyrocompass itself presents some systematic errors. In the implementation, these errors are described by a Gaussian error. This is, however, not a proper error model for the gyrocompass. Thus, the error behavior of gyrocompass is not well identified by the integration. This will be an essential part of further work. As the heading accuracy is dominated by the gyrocompass in such integration architecture, these systematic errors will also bias the heading results from the integration. It is therefore recommended to bring the GNSS-compass also into the integration.

If the attitude results from GNSS-compass are appended to the measurement vector, the attitude results will be fully observable and directly aided by the GNSS-compass. The attitude difference between the GNSS-compass and the integration are presented in Figure 11.

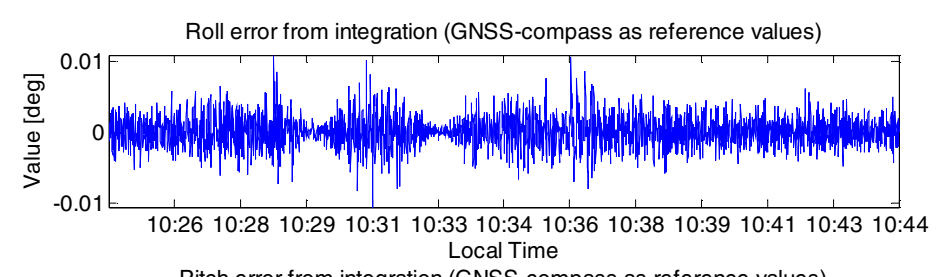

Pitch error from integration (GNSS-compass as reference values)

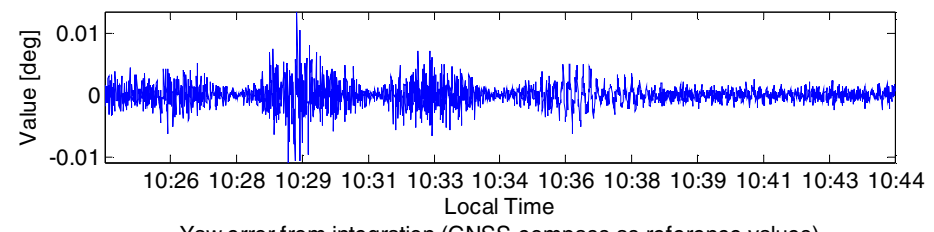

Yaw error from integration (GNSS-compass as reference values)

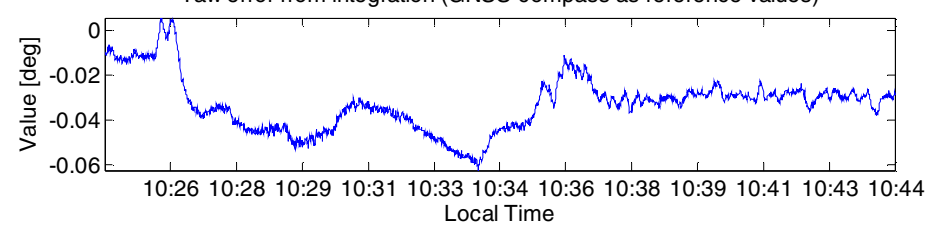

Figure 11 Attitude errors GNSS/IMU/Gyrocompass/GNSS-compass integration

It can be seen that, due to the fusion with GNSS-compass, the attitude accuracy will be dominated by the GNSS-compass. This integration, however, needs a validation of the quality of GNSS-compass in order to guarantee the validity of assigned $a$ priori error. From the similar error behavior of yaw angle given in Figure 10 and Figure 11 it can be seen that, the uncompensated gyrocompass error still has negative influence on the results from the integration.

The integration with IMU can also improve the performance of GNSS-compass. At one hand, the GNSScompass is subject to the quality of GNSS signals, and hence it might not work if GNSS-signal is of low quality, at loss of signal tracking or no solution of carrier phase ambiguity. The IMU provides high quality measurement of attitude dynamics for short-term, and hence it allows a high-accuracy bridge during the failure of GNSS-compass. At the other hand, depending on the baseline length, the GNSS-compass might reach the accuracy at about 0.1 degrees or less than that. Although this already fulfills the accuracy requirement of attitude for non-critical maritime navigation, the IMU might provide more precise attitude dynamics. Figure 12 shows the epoch-to-epoch attitude variation estimated by the GNSScompass and by the IMU strapdown processing in a short-term. 

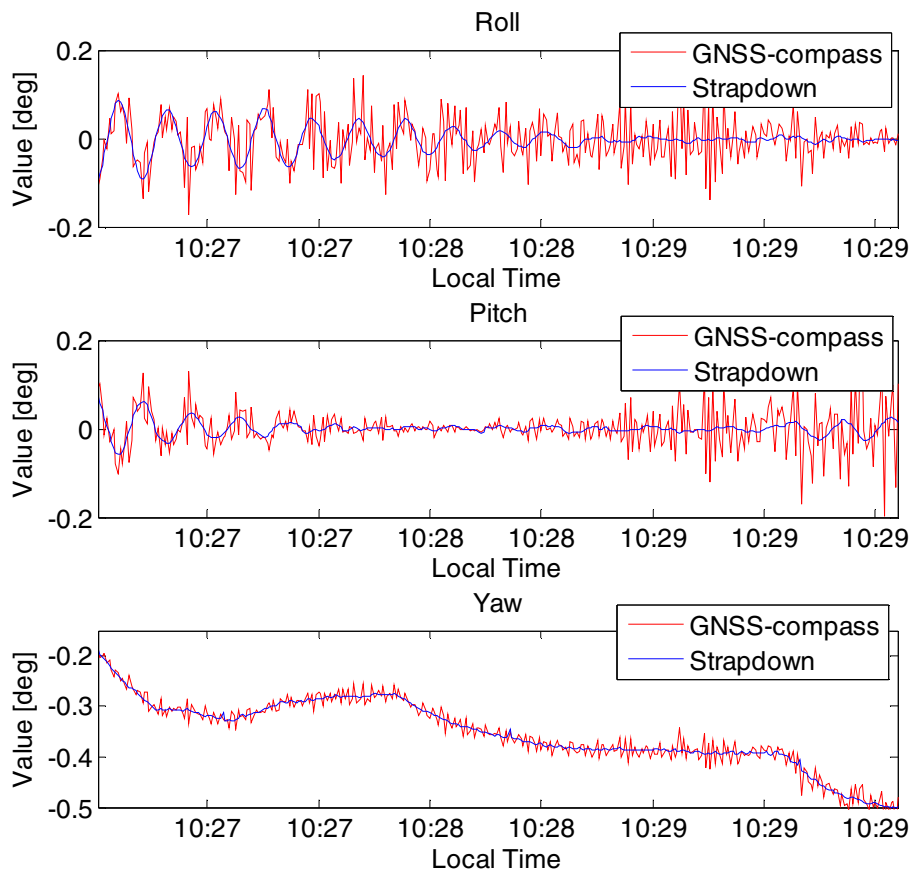

Figure 12 Epoch-for-epoch attitude variation estimated by GNSS-compass and IMU strapdown

It can be seen that the IMU show results with less noise compared to GNSS-compass. This agrees with the limited maneuverability of the vessel. For this point of view, the IMU helps with the improvement of attitude accuracy.

\section{CONCLUSION}

This paper discusses the performance of different existing maritime north-finding sensors. Based on that, the concept of DLR maritime PNT Unit is introduced. With the PNT Unit, GNSS, inertial sensors, GNSS-compass and gyrocompass can be fused to improve the overall performance of heading determination and moreover, to assess the accuracy. Some preliminary results of a measurement campaign are presented to show the improvement in terms of accuracy and continuity using the sensor-fusion architecture. At one hand, the addition of GNSS-compass could improve the observability of attitude angles and compensate the systematic error of gyrocompass. At the other hand, the use of IMU can also improve the attitude accuracy and furthermore serves as a high-accuracy backup system to improve the continuity during the outage of other sensors.

Another improvement brought by the PNT Unit is the realization of quantified error estimation of PNT parameters as well as the individual sensor data. This aspect will be discussed in further papers.

\section{ACKNOWLEDGMENT}

The authors would like to thank the Federal Maritime and Hydrographic Agency of Germany (BSH), in particular Mr. Tobias Ehlers and the crew of the DENEB with their captain
Mr. Gentes for the support during the measurement activities. The authors are also grateful for the assistance of their colleagues Pawel Banys, Carsten Becker, Stefan Gewies, Uwe Netzband and Thoralf Noack.

\section{REFERENCES}

[1] IMO, "NAV 53/13 Development of an e-Navigation Strategy," 2007.

[2] IMO, "NAV 55/WP.5 Development of an e-Navigation Strategy Implementation Plan," NAV55/ WP5, 2009.

[3] IMO, "SOLAS CHAPTER V: SAFETY OF NAVIGATION," 2002.

[4] IMO, "Resolution MSC.252(83): Adoption of the Revised Performance Standards for Integrated Navigation Systems (INS)," 2007.

[5] IMO, "Resolution MSC.252(83): Adoption of the Revised Performance Standards for Integrated Navigation Systems (INS)," 2007.

[6] R. Ziebold, Z. Dai, T. Noack, and E. Engler, "Concept for an Integrated PNT-Unit for Maritime Applications," Proceedings of the 5th ESA Workshop on Satellite Navigation Technologies. Navitec 2010, 8.-10. Dec. 2010, Noordwijk, The Netherlands., 2010.

[7] B. Hoffman-Wellenhof, K. Legat, and M. Wieser, Navigation: Principle of Positioning and Guidance. 2003.

[8] S. Schlüter, J. Beckheinrich, A. Hirrle, T. Noack, D. Klähn, R. Reimer, C. Becker, and E. Engler, "ALEGRO - A step towards maritime Ground Based Augmentation Systems," presented at the Marine Traffic Engineering Conference, Szczecin, Poland, 2009.

[9] P. D. Groves, Principles of GNSS, Inertial, and Multi-Sensor Integrated Navigation Systems (GNSS Technology and Applications). Artech House Publishers, 2007.

[10] N. Bowditch, The American Practical Navigator. National imagery and mapping agency, 2002.

[11] D. Simon, Optimal State Estimation. John Wiley \& Sons, Inc., 2006. 